\title{
Compensation of gravity on cold atoms by a linear optical potential
}

\author{
Kosuke Shibata $\odot,{ }^{*}$ Hidehiko Ikeda, Ryota Suzuki, and Takuya Hirano $\odot$ \\ Department of Physics, Gakushuin University Tokyo, Japan
}

(Received 1 August 2019; published 23 January 2020)

\begin{abstract}
We demonstrate gravity compensation for an ultracold gas of ${ }^{87} \mathrm{Rb}$ atoms with a time-averaged optical potential. The position of a far-off-resonance beam is temporally modulated with an acousto-optic deflector to efficiently produce a potential with a linear gradient independent of the atomic magnetic sublevels. We realize compensation of the gravity sag and preparation of a degenerate gas in a trap with weak vertical confinement. Optical gravity compensation will provide the opportunity to perform experiments under microgravity in a laboratory and broaden the scope of cold atom research.
\end{abstract}

DOI: 10.1103/PhysRevResearch.2.013068

\section{INTRODUCTION}

Cold atoms are significantly affected by gravity because of their low kinetic energy. While cold atoms can be used to measure fundamental constants such as the Newtonian gravitational constant [1] and offer interesting subjects such as the gravity on a quantum gas, gravity often induces undesired effects in experiments. This limits the size, shape, and density of a trapped atom cloud because of the requirement to vertically hold atoms against gravity. It also deteriorates the performance of precision measurements using atoms, e.g., in atomic interferometry [2].

A microgravity environment, free of these limitations, offers a rich ground for science and applications. Microgravity environments have been realized in ballistic flight [3], tall drop towers [4,5], a specially designed elevator [6], and space [7]. Orbital microgravity [8] gives new opportunities to investigate exotic quantum phenomena with essentially unlimited duration $[9,10]$. A magnetic field gradient is a convenient tool for gravity cancellation on atoms in a magnetically sensitive atomic state. Magnetic gravity cancellation has been used for producing dilute Bose-Einstein condensates (BEC) below 500 pK [11], a slow atom laser [12], and a homogeneous three-dimensional (3D) gas [13].

The production of microgravity environments, regardless of the atomic states, will broaden the scope of experiments in laboratories. As an important application, dilute or homogeneous gases with spin degrees of freedom can be produced. A homogeneous spinor gas is a good platform for precise magnetometry because of the absence of density-dependent inhomogeneous collisional shifts [14]. It is also useful for a precise matter-wave interferometer [15].

The optical potential due to a far-detuned light field is state independent [16] and should be a promising component for

\footnotetext{
*shibata@qo.phys.gakushuin.ac.jp

Published by the American Physical Society under the terms of the Creative Commons Attribution 4.0 International license. Further distribution of this work must maintain attribution to the author(s) and the published article's title, journal citation, and DOI.
}

convenient and versatile gravity cancellation. Optical gravity cancellation to achieve a microgravity environment has not been realized, however. A difficulty for optical gravity cancellation is creating a sufficiently linear intensity gradient over an atom cloud (typically $10^{2}$ to $10^{3} \mu \mathrm{m}$ ). With a Gaussian beam, gravity cancellation for an entire gas requires a beam of very large size and an impractically enormous power, although the reduction of a gravity sag has been implemented $[17,18]$. Spatial light modulators, which have recently been widely used in cold atom experiments to produce optical traps with various configurations $[13,19,20]$, can in principle create a linear intensity profile. However, it might be a challenge to achieve a beam profile using a spatial light modulator of good linearity over a wide area covering an atom cloud.

Here, we propose gravity cancellation by a time-averaged linear optical potential, produced by deflecting a beam with an acousto-optic deflector (AOD) driven by a frequencymodulated radio-frequency (rf) wave. Such a "painting" technique [21] has been used for the construction of integrated coherent matter wave circuits [22] and the production of dynamically shaped atom traps for rapid evaporative cooling [23]. As we show below, a beam having a linear intensity gradient suitable for gravity compensation can be produced with the painting technique. We apply a beam with a linear intensity gradient to compensate for the gravity sag of an ultracold ${ }^{87} \mathrm{Rb}$ atom gas in an optical trap. Furthermore, we prepare a degenerate gas in a trap with weak vertical confinement with the aid of the compensation beam.

The paper is organized as follows. We explain the method for producing an intensity profile with a linear gradient by the painting technique in Sec. II. In Sec. III, we describe the experimental results for the produced intensity profiles and the gravity compensation on cold atom clouds. The potential applications are discussed in Sec. IV. We conclude the paper in Sec. V.

\section{METHOD}

Before presenting the experimental details, we describe how to design a target intensity profile. The scheme relies on the simple fact that the more slowly (more quickly) the beam 
crosses a certain position, the larger (smaller) the light intensity at the position. We design the intensity profile through frequency modulation (FM) of the rf wave applied to the AOD. We only modulate the rf frequency to efficiently use the beam power, whereas amplitude modulation allows more flexible generation of a desired profile.

Let us consider the time-averaged intensity produced by a beam scanned along the $y$ direction. We first consider the case in which the beam size is sufficiently small. Its position at time $t$ is designated by $y(t)$ and the intensity distribution at $t$ is given by $I(y, t)=I_{0} \delta(y-y(t))$. In this case, the intensity averaged from $t=0$ to $t=T, \bar{I}(y) \equiv \frac{1}{T} \int_{0}^{T} I(y, t) d t$, is inversely proportional to $|\dot{y}(t)|$. The trajectory $y(t)$ giving a target averaged intensity $\bar{I}(y)$ is in general given by the solution of

$$
\frac{C}{\dot{y}(t)}=\bar{I}(y)
$$

with $C$ being a constant. Therefore, a linearly increasing intensity is produced with a trajectory satisfying $y(t)-y(0) \propto \sqrt{t}$. When $y(t)$ responds linearly to the rf frequency (which holds for a small beam displacement), the FM wave form $v(t)$ to produce a linear intensity profile is given by

$$
v(t)=b \sqrt{t}+v(0)
$$

where $b$ is a constant.

We can produce a profile with a linear slope by frequency modulation using Eq. (2) with a Gaussian beam with a finite beam waist $w$. The averaged intensity of the beam with its center scanned from $y(0)$ to $y(T)=y(0)+L$ according to Eq. (2) is written as

$$
\begin{aligned}
\frac{\bar{I}(y)}{I_{0}}= & \sqrt{\pi} \beta \eta\left[\operatorname{erf}\left(\frac{1-\eta}{\beta}\right)+\operatorname{erf}\left(\frac{\eta}{\beta}\right)\right] \\
& +\beta^{2}\left[e^{-\eta^{2} / \beta^{2}}-e^{-(1-\eta)^{2} / \beta^{2}}\right],
\end{aligned}
$$

where $I_{0}$ is the peak beam intensity without modulation, $\eta=\frac{y}{L}, \beta=\frac{w}{\sqrt{2} L}$, and $\operatorname{erf}(z)=\frac{2}{\sqrt{\pi}} \int_{0}^{z} e^{-t^{2}} d t$. As $\beta$ becomes smaller, better linearity is achieved. In other words, a linear intensity gradient is produced when the scan width $L$ is sufficiently larger than $w$. With a scan width of $L=7 w$, the deviation from a perfect linear intensity is smaller than $10^{-6}$ over a range of $3 w$. When $L=20 w$, the relative deviation can be smaller than $10^{-14}$ over a range of $13 \mathrm{w}$.

The experimental setup for the optical painting is shown in Fig. 1(a). The compensation beam source is a fiber Bragg grating $(\mathrm{FBG})$ stabilized laser with a center wavelength of $\lambda=976 \mathrm{~nm}$ (Thorlabs, BL976-PAG900), producing an attractive potential for $\mathrm{Rb}$ atoms. The beam is deflected by a two-axis AOD (IntraAction Corp., DTD-274HD6M). An rf wave form with its frequency modulated according to Eq. (2) is repeatedly generated by an arbitrary wave-form generator (Lecroy, ArbStudio 1102) and sent to the AOD through a mixer for light power adjustment and an $\mathrm{rf}$ amplifier. The deflected beam is focused by a lens of focal length $100 \mathrm{~mm}$ after the AOD and is demagnified onto the target plane in a vacuum glass cell [ $\Sigma_{2}$ in Fig. 1(a)] through a pair of lenses with focal lengths of 500 and $200 \mathrm{~mm}$.

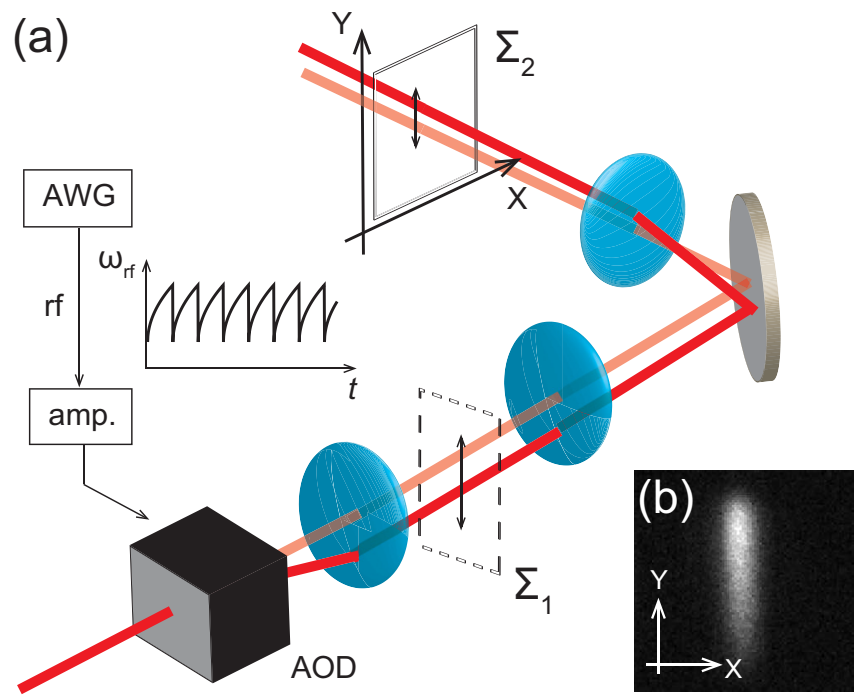

FIG. 1. (a) Setup for optical painting. A frequency modulated rf wave from an arbitrary wave-form generator (AWG) is sent to the AOD. The beam deflected by the AOD is focused by a lens on the plane $\Sigma_{1}$. The deflected beam displacement at $\Sigma_{1}$ is proportional to the change in the rf frequency. The beam is demagnified onto the target plane $\Sigma_{2}$ in a vacuum glass cell (not shown) using a lens pair. (b) An example 2D intensity profile of a painted beam. The field of view is $200 \times 200 \mu \mathrm{m}$.

\section{EXPERIMENTAL RESULTS}

We examine the time-averaged intensity of the scanned beam to confirm the validity of the method and to evaluate the performance of the experimental system. We scan the rf frequency on the AOD for vertical beam position scan according to

$$
v(t)=v_{0}+\Delta v \sqrt{\frac{t}{T}},
$$

where $T=100 \mu$ s is the scan cycle. We take images of the beam using an imaging system consisting of lenses and a charge-coupled device (CCD) camera after the cell. The camera exposure time is set to $20 \mathrm{~ms}$, sufficiently longer than $T$, to measure the effective average intensity profile. A typical measured 2D intensity profile with $\Delta v=2 \mathrm{MHz}$ is shown in Fig. 1(b). The vertical intensity distributions obtained by integrating the $2 \mathrm{D}$ profile along the horizontal $(X)$ axis are plotted in Fig. 2(a). The observed profiles with frequency scan widths of $\Delta v=1,2,3$, and $4 \mathrm{MHz}$ are in good agreement with the calculated profiles based on Eq. (3) shown in Fig. 2(b).

The slope regions are fitted by a linear function to determine the gradient [see Fig. 2(c)]. We observe no significant systematic deviation from the linear fit, as shown in Fig. 2(d), implying good linearity of the beam intensity. The fitting residuals mainly come from noise in the imaging. The intensity gradient per unit power, $\gamma$, is plotted as a function of the scan width in Fig. 2(e). We fit the gradient by $a \Delta v^{-2}$, the validity of which we confirm with the calculated profiles, obtaining $a=7.49(16) \mathrm{mW} \mathrm{mm}^{-3}(\mathrm{MHz})^{-2}$.

The above intensity profiles are actually not very suitable for compensating gravity on a cold atom cloud, because the 


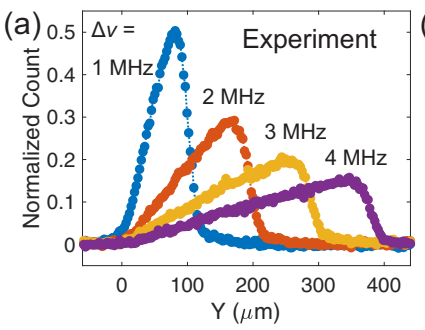

(c)

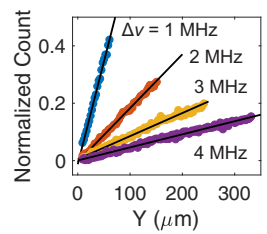

(d)
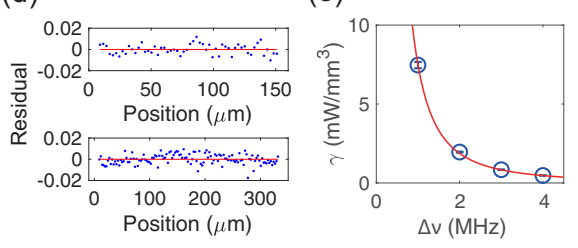

FIG. 2. Horizontally integrated intensity of the painted beam. (a) Experimental results. Data with frequency scan widths of 1, 2, 3 , and $4 \mathrm{MHz}$ are represented by the blue, orange, yellow, and purple points, respectively. The intensity is normalized by the peak intensity without modulation. (b) Calculated intensity. The calculation for the scan widths $(3.5,7,10.5,14) w$ are represented by the blue, orange, yellow, and purple lines, respectively, where $w$ is the beam waist and measured to be 24.7(7) $\mu \mathrm{m}$. The vertical dotted lines indicate the boundaries of the scanned beam center for a scan width of $3.5 \mathrm{w}$. (c) Linear fits to the slope regions of measured profiles. (d) Residuals of the linear fits for scan widths of $2 \mathrm{MHz}$ (upper panel) and $4 \mathrm{MHz}$ (lower panel). (e) Relation between the intensity gradient $\gamma$ and the frequency scan width $\Delta v$. The red solid curve is a fit using $a \Delta v^{-2}$ (see the text).

potential is horizontally inhomogeneous. As a countermeasure, we sweep the rf frequency on the horizontal AOD axis linearly in addition to the vertical modulation to realize a top-flat horizontal beam profile. To be specific, we apply horizontal and vertical frequency modulations given by

$$
\begin{aligned}
& v_{x}(t)=v_{x 0}+\Delta v_{x} \frac{t}{T_{x}}, \\
& v_{y}(t)=v_{y 0}+\Delta v_{y} \sqrt{\frac{t}{T_{y}}},
\end{aligned}
$$

respectively. We set $T_{x}=8 \mu \mathrm{s}$ and $T_{y}=250 \mu \mathrm{s}$ to satisfy $T_{x} \ll T_{y}$, keeping the repetition rates $T_{x}^{-1}$ and $T_{y}^{-1}$ much higher than the trap frequencies.

Figure 3(c) shows the intensity profile for frequency modulation with $\Delta v_{x}=640 \mathrm{kHz}$ and $\Delta v_{y}=1.5 \mathrm{MHz}$. Vertically integrated intensity distributions with different $\Delta v_{x}$ are shown in Fig. 3(a). The corresponding calculated profiles are given by

$$
\tilde{I}(X)=\frac{\sqrt{\pi} \tilde{I}(0)}{4 \alpha}[\operatorname{erf}(\chi+\alpha)-\operatorname{erf}(\chi-\alpha)],
$$

where $\chi=\sqrt{2} X / w$ and $\alpha=L_{X} /(\sqrt{8} w)$ with $L_{X}$ being the horizontal scan width, and are plotted in Fig. 3(b), showing good agreement with the experimental results. When $\Delta v_{x}=$ $0.64 \mathrm{MHz}$, the intensity inhomogeneity in $|X|<3 \mu \mathrm{m}$ is reduced to less than $10^{-2}$, at the cost of a decrease of the peak intensity by $1 / 3$, as shown in Fig. 3(d). The horizontal scan works better when the horizontal scan width becomes large
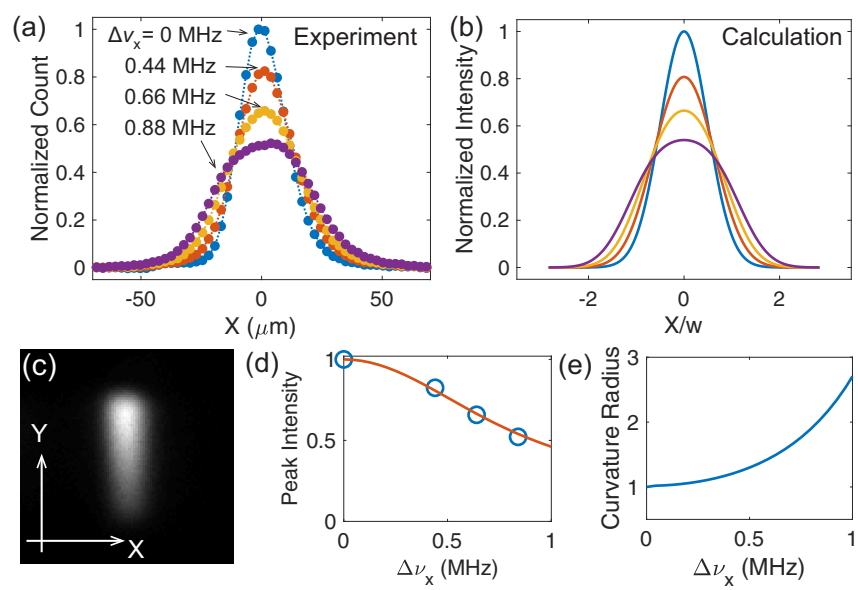

FIG. 3. Vertically integrated intensity of the painted beam. (a) Experimental results. The vertically integrated intensities with $\Delta v_{x}=0,0.44,0.64$, and $0.84 \mathrm{MHz}$ are represented by the blue, orange, yellow, and purple points, respectively. (b) Calculated intensity. The calculations for horizontal scan widths $(0,1.23,1.79,2.35) w$ are represented by the black, blue, green, and red solid lines, respectively. (c) $2 \mathrm{D}$ intensity profile with $\Delta v_{x}=0.64 \mathrm{MHz}$. The field of view is $200 \times 200 \mu \mathrm{m}$. (d) Measured peak intensity (blue circles) and calculated peak intensity (red solid line) as a function of $\Delta v_{x}$. (e) Calculated curvature radius of the beam as a function of $\Delta v_{x}$. The radius is normalized by the value without scan.

compared with the original beam width, as can be guessed from the beam profiles in Figs. 3(a) and 3(b). For a measure of the homogeneity of the scanned profile, we present a calculated beam curvature radius in Fig. 3(e).

We apply the painted beam to a cold atom cloud in a crossed optical trap [24]. The atoms are transferred from a magnetic trap to the optical trap and are cooled by forced evaporative cooling to degeneracy. We prepare a partially Bose-condensed gas of $3 \times 10^{5}$ atoms in the $|F, m\rangle=|2,2\rangle$ state. The trap frequencies of the crossed optical trap are $\left(\omega_{x}, \omega_{y}, \omega_{z}\right)=2 \pi \times(340,70,30) \mathrm{Hz}$. After the evaporation, we ramp up the compensation beam power, $P$, during a period of $100 \mathrm{~ms}$, and hold the atoms for another $100 \mathrm{~ms}$. Then, we release the atoms from the trap and take an absorption image after a time of flight (TOF). The beam power is stabilized and the power fluctuation is below $3 \times 10^{-3}$.

We first present results for scan widths of $\Delta v_{x}=0.64 \mathrm{MHz}$ and $\Delta v_{y}=1.5 \mathrm{MHz}$. The atoms move upward by the application of the compensation beam, as can be seen by comparing the cases with $P=2 \mathrm{~mW}$ [Fig. 4(a)] and $116 \mathrm{~mW}$ [Fig. 4(b)]. The absorption images in Figs. 4(a)-4(c) are taken with a TOF of $0.2 \mathrm{~ms}$. These images give essentially the in situ density profile of the atom cloud because atoms fall only $0.2 \mu \mathrm{m}$ and expansion of the gas is negligible during $0.2 \mathrm{~ms}$. We plot the vertical displacement of the cloud center, $\Delta Y$, in Fig. 4(d) (blue circles). The position for the case with $P=2 \mathrm{~mW}$ is taken as the origin of the displacement. While the displacement is smaller than or comparable to the vertical waist size of the trap beam $(\approx 70 \mu \mathrm{m})$, the original potential can be approximated by a parabola and the linear compensation beam should lead to a displacement proportional to $P$. The observed displacement is consistent with the fact that gravity sag is 


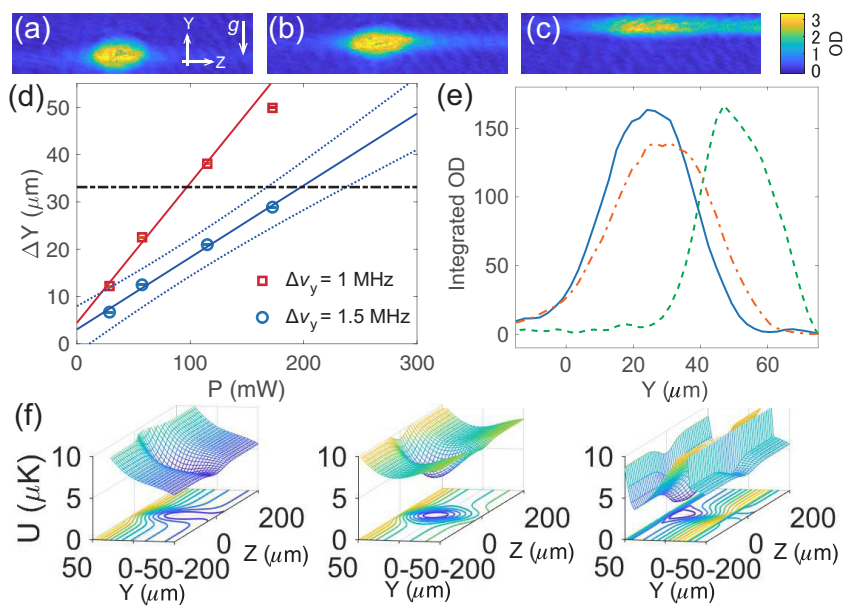

FIG. 4. Gravity compensation in the optical potential. Absorption images of the atom clouds after a TOF of $0.2 \mathrm{~ms}$ (a) with almost no compensation, (b) with a compensation beam with $P=$ $116 \mathrm{~mW}$ and $\Delta v_{y}=1.5 \mathrm{MHz}$, and (c) with a beam with $P=$ $174 \mathrm{~mW}$ and $\Delta v_{y}=1 \mathrm{MHz}$. The field of view in each panel is $100 \times 400 \mu \mathrm{m}$. (d) Displacement of the vertical cloud center. The data with $\Delta v_{y}=1$ and $1.5 \mathrm{MHz}$ are represented by the red squares and blue circles, respectively. The horizontal dash-dotted line represent predicted displacement with gravity cancellation, $\Delta Y_{0}=$ $33 \mu \mathrm{m}$. The solid red and blue lines are a linear fit to the data with $\Delta v_{y}=1$ and $1.5 \mathrm{MHz}$, respectively. The dotted blue lines represent the prediction bounds with confidence level of $95 \%$ for the latter fit. (e) Horizontally integrated optical densities of panels (b) [blue solid line] and (c) [green dashed line]. The orange dash-dotted line represents the density for a weakened trap in Fig. 5(a). (f) Calculated potential in the $Y-Z$ plane of $100 \times 400 \mu \mathrm{m}$. The left, middle, and right panels correspond to the data in panels (a)-(c), respectively. The potential contour is plotted at the bottom in each panel.

compensated by the linear optical potential. The displacement in the case of the perfect gravity cancellation is estimated as $\Delta Y_{0}=33 \mu \mathrm{m}$. With this estimation, we conclude that we are capable of full gravity cancellation with these scan widths and an available beam power.

The results with $\Delta v_{y}=1 \mathrm{MHz}$, which produces a steeper intensity gradient, are also plotted in Fig. 4(d) (red squares). The displacement is larger than the case with $\Delta v_{y}=1.5 \mathrm{MHz}$, as expected, but tends to saturate for higher $P$. This is because the compensation is so great that the atoms are pulled up to the upper boundary of the painted potential. In fact, when a beam with $P=174 \mathrm{~mW}$ is applied, the vertical width of the atom cloud becomes narrow, as can be seen in Figs. 4(c) and 4(e), resulting from the combined potential of gravity and the painted beam. At the same time, the atom distribution is elongated along $Z$ axis. The elongated atom distribution is further evidence that the beam power is sufficient for gravity compensation, because the vertical confinement in the side region is provided by a single axial optical trap and cannot trap atoms without gravity compensation.

The observed atom distributions are reasonably consistent with calculated potential, shown in Fig. 4(f). In the situation in Fig. 4(c) [right panel in Fig. 4(f)], gravity is overcompensated and the atom cloud is located above the center of the crossed optical trap potential. This leads to weak $Z$ confinement,
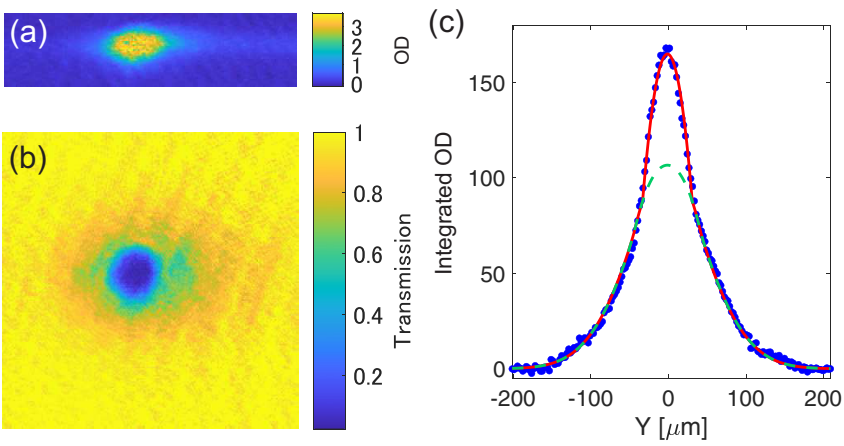

FIG. 5. Absorption images with TOFs of (a) $0.2 \mathrm{~ms}$ and (b) $16 \mathrm{~ms}$. (c) Horizontally integrated optical density of the data shown in panel (b). The red solid and green dashed lines are fitting curves using a bimodal function and its thermal component, respectively.

which is originally provided by the radial beam propagating along the $X$ axis. As a result, the atom cloud broadens along the $Z$ axis. The asymmetry along the $Z$ axis observed in the experiment is ascribed to slight misalignment of the trap beams.

We estimate the power required for gravity cancellation on the basis of $\Delta Y_{0}$ and the linear extrapolation of the data for $\Delta v_{y}=1.5 \mathrm{MHz}$ as $198_{-30}^{+39} \mathrm{~mW}$, where the subscripts denote the confidence interval obtained from the crossing points of the prediction curves of $95 \%$ confidence levels and the line at $\Delta Y=\Delta Y_{0}$. On the other hand, the power for gravity cancellation is expected to be $260 \mathrm{~mW}$ using the result of the intensity profile measurement. We perform similar analysis for the data with $\Delta v_{y}=1 \mathrm{MHz}$. A linear fit to the data [red solid line in Fig. 4(d)] crosses $\Delta Y_{0}$ at $P=97 \mathrm{~mW}$. We note that the data with overcompensation $(P=174 \mathrm{~mW})$ is excluded from the fit. The power for gravity cancellation is again smaller than the expected power of $116 \mathrm{~mW}$ on the basis of the profile measurement. The discrepancy in the required power may be due to an error in the calibration of the magnification of the beam profile analysis system, $M$, which largely influences the estimation of the intensity gradient (approximately $\propto M^{5}$ ). Another possible cause is the aberration in the beam analysis system, leading to the overestimate of the beam size.

If gravity is properly canceled, the atoms can be held in a trap with weak vertical confinement. We demonstrate the expansion of atoms into a weaker confinement with the aid of a compensation beam. In this experiment, we set the scan widths to $\Delta v_{x}=0.8 \mathrm{MHz}$ and $\Delta v_{y}=1.3 \mathrm{MHz}$. During the ramp up of the compensation beam over $300 \mathrm{~ms}$, we decrease the axial beam power from 131 to $29 \mathrm{~mW}$ and the radial beam power from 58 to $38 \mathrm{~mW}$. The mean trap frequency of the weakened trap is estimated from the total number of atoms and the critical temperature to be $2 \pi \times 59 \mathrm{~Hz}, \approx 2 / 3$ of the original mean trap frequency.

While the crossed optical trap with decreased power alone cannot hold atoms because of the severe potential distortion due to gravity, we observe that the atoms remain after a hold time of $600 \mathrm{~ms}$ in the weakened optical trap with the compensation beam. An image obtained with a TOF of $0.2 \mathrm{~ms}$ and $P=200 \mathrm{~mW}$ is shown in Fig. 5(a). The vertical cloud size 
becomes slightly larger [see Fig. 4(e)], reflecting the weaker vertical confinement. The gas is a partially degenerate BEC. By a bimodal fitting to the image with a TOF of $16 \mathrm{~ms}$ [see Figs. 5(b) and 5(c)], we obtain a total number of atoms of $2.8 \times 10^{5}$ and a condensate fraction of 0.29 . The measured temperature is $T=142 \pm 7 \mathrm{nK}$, The result suggests that the painted linear potential does not cause severe heating of the atoms [25]. We estimate the heating rate as $100 \mathrm{nK} / \mathrm{s}$, neglecting the temperature change due to adiabatic decompression from the original trap to the final trap. Although this rate is higher than the expected heating rate of $70 \mathrm{nK} / \mathrm{s}$ due to the photon scattering from the trap beams, it is not too significant to prevent performing experiments with a BEC. We can further decrease the trap beam power to produce a BEC with no discernible thermal component.

\section{DISCUSSION}

The demonstrated optical gravity compensation will broaden the scope of cold atom research. Cold atom gases can be prepared in weak or homogeneous traps regardless of the spin. This opens the possibility of preparing gases of various spin components in weak or homogeneous traps, including homogeneous spinor gases. Gravity compensation for spinless alkaline-earth(-like) atoms can also be realized.

The method may be applied to construct compact precise interferometers requiring a long-time expansion of atoms. As a matter of fact, if one attempts to attain a very large compensation volume, the beam inhomogeneity along the compensation beam propagation axis becomes troublesome. For example, a laser with a power of $7 \mathrm{~W}$ and a waist of $50 \mu \mathrm{m}$ at a wavelength of $808 \mathrm{~nm}$ should compensate gravity on $\mathrm{Rb}$ atoms over a volume of $0.4 \times 0.4 \times 0.4 \mathrm{~mm}$, with the vertical potential gradient varying by $2 \%$ along the beam axis. A naive solution is to use a beam of a large waist (therefore, with a large Rayleigh range) with increasing scan width to keep a good vertical linearity. The compensation volume or the linearity should be compromised depending on the available beam power. For precise measurements, the use of a compensation beam at a magic wavelength would be required and the gravity compensation volume may be reduced due to the limited beam power at the magic wavelength. The limitation, however, might be relaxed with a more sophisticated configuration such as crossed beams for painting and/or improvements in laser technology. The reduction of the axial inhomogeneity will enable free expansion in a microgravity environment over several hundreds of milliseconds, comparable to that in experiments in tall towers [4,5] and space [7].

\section{CONCLUSION}

We demonstrate compensation of gravity on cold atoms with a time-averaged linear optical potential. The optical gravity cancellation will open new possibilities for cold atom experiments, including the production of very dilute gases, the study of homogeneous gases with spin degrees of freedom, and precise measurements.

\section{ACKNOWLEDGMENTS}

This work was supported by MEXT Quantum Leap Flagship Program (MEXT Q-LEAP) Grant No. JPMXS0118070326, the Asahi Glass Foundation, and JSPS KAKENHI Grant No. JP18K45678. We would like to thank Munekazu Horikoshi for his inspiring comments on the applications of this work.
[1] G. Rosi, F. Sorrentino, L. Cacciapuoti, M. Prevedelli, and G. M. Tino, Nature (London) 510, 518 (2014).

[2] G. D'Amico, G. Rosi, S. Zhan, L. Cacciapuoti, M. Fattori, and G. M. Tino, Phys. Rev. Lett. 119, 253201 (2017).

[3] G. Stern, B. Battelier, and R. Geiger, Eur. Phys. J. D 53, 353 (2009).

[4] T. van Zoest, N. Gaaloul, Y. Singh, H. Ahlers, W. Herr, S. T. Seidel, W. Ertmer, E. Rasel, M. Eckart, E. Kajari, S. Arnold, G. Nandi, W. P. Schleich, R. Walser, A. Vogel, K. Sengstock, K. Bongs, W. Lewoczko-Adamczyk, M. Schiemangk, T. Schuldt, A. Peters, T. Könemann, H. Müntinga, C. Lämmerzahl, H. Dittus, T. Steinmetz, T. W. Hänsch, and J. Reichel, Science 328, 1540 (2010).

[5] H. Müntinga, H. Ahlers, M. Krutzik, A. Wenzlawski, S. Arnold, D. Becker, K. Bongs, H. Dittus, H. Duncker, N. Gaaloul, C. Gherasim, E. Giese, C. Grzeschik, T. W. Hänsch, O. Hellmig, W. Herr, S. Herrmann, E. Kajari, S. Kleinert, C. Lämmerzahl, W. Lewoczko-Adamczyk, J. Malcolm, N. Meyer, R. Nolte, A. Peters, M. Popp, J. Reichel, A. Roura, J. Rudolph, M. Schiemangk, M. Schneider, S. T. Seidel, K. Sengstock, V. Tamma, T. Valenzuela, A. Vogel, R. Walser, T. Wendrich, P. Windpassinger, W. Zeller, T. van Zoest, W. Ertmer, W. P. Schleich, and E. M. Rasel, Phys. Rev. Lett. 110, 093602 (2013).
[6] G. Condon, M. Rabault, B. Barrett, L. Chichet, R. Arguel, H. Eneriz-Imaz, D. Naik, A. Bertoldi, B. Battelier, A. Landragin, and P. Bouyer, Phys. Rev. Lett. 123, 240402 (2019).

[7] D. Becker, M. D. Lachmann, S. T. Seidel, H. Ahlers, A. N. Dinkelaker, J. Grosse, O. Hellmig, H. Müntinga, V. Schkolnik, T. Wendrich, A. Wenzlawski, B. Weps, R. Corgier, T. Franz, N. Gaaloul, W. Herr, D. Lüdtke, M. Popp, S. Amri, H. Duncker, M. Erbe, A. Kohfeldt, A. Kubelka-Lange, C. Braxmaier, E. Charron, W. Ertmer, M. Krutzik, C. Lämmerzahl, A. Peters, W. P. Schleich, K. Sengstock, R. Walser, A. Wicht, P. Windpassinger, and E. M. Rasel, Nature (London) 562, 391 (2018).

[8] E. R. Elliott, M. C. Krutzik, J. R. Williams, R. J. Thompson, and D. C. Aveline, npj Microgravity 4, 16 (2018).

[9] C. A. Sackett, T. C. Lam, J. C. Stickney, and J. H. Burke, Microgravity Sci. Technol. 30, 155 (2018).

[10] N. Lundblad, R. A. Carollo, C. Lannert, M. J. Gold, X. Jiang, D. Paseltiner, N. Sergay, and D. C. Aveline, npj Microgravity 5, 30 (2019).

[11] A. E. Leanhardt, T. A. Pasquini, M. Saba, A. Schirotzek, Y. Shin, D. Kielpinski, D. E. Pritchard, and W. Ketterle, Science 301, 1513 (2003). 
[12] G. K. Buning, J. Will, W. Ertmer, C. Klempt, and J. Arlt, Appl. Phys. B 100, 117 (2010).

[13] A. L. Gaunt, T. F. Schmidutz, I. Gotlibovych, R. P. Smith, and Z. Hadzibabic, Phys. Rev. Lett. 110, 200406 (2013).

[14] D. M. Harber, H. J. Lewandowski, J. M. McGuirk, and E. A. Cornell, Phys. Rev. A 66, 053616 (2002).

[15] A. D. Cronin, J. Schmiedmayer, and D. E. Pritchard, Rev. Mod. Phys. 81, 1051 (2009).

[16] R. Grimm, M. Weidemüller, and Y. B. Ovchinnikov, Adv. At. Mol. Opt. Phys. 42, 95 (2000).

[17] H. Konishi, F. Schafer, S. Ueda, and Y. Takahashi, New J. Phys. 18, 103009 (2016).

[18] D. S. Naik, G. Kuyumjyan, D. Pandey, P. Bouyer, and A. Bertoldi, Quantum Sci. Technol. 3, 045009 (2018).
[19] F. Nogrette, H. Labuhn, S. Ravets, D. Barredo, L. Béguin, A. Vernier, T. Lahaye, and A. Browaeys, Phys. Rev. X 4, 021034 (2014).

[20] G. Gauthier, I. Lenton, N. M. Parry, M. Baker, M. J. Davis, H. Rubinsztein-Dunlop, and T. W. Neely, Optica 3, 1136 (2016).

[21] K. Henderson, C. Ryu, C. MacCormick, and M. G. Boshier, New J. Phys. 11, 043030 (2009).

[22] C. Ryu and M. G. Boshier, New J. Phys. 17, 092002 (2015).

[23] R. Roy, A. Green, R. Bowler, and S. Gupta, Phys. Rev. A 93, 043403 (2016).

[24] K. Shibata, A. Torii, H. Shibayama, Y. Eto, H. Saito, and T. Hirano, Phys. Rev. A 99, 013622 (2019).

[25] R. Onofrio, D. S. Durfee, C. Raman, M. Köhl, C. E. Kuklewicz, and W. Ketterle, Phys. Rev. Lett. 84, 810 (2000). 